\title{
The impact of cardiac CT on the appropriate utilization of catheter coronary angiography
}

\author{
Wagdi, P ; Alkadhi, H
}

\begin{abstract}
The purpose of this study was to evaluate the impact of computed tomography coronary angiography (CTCA) on the appropriate utilization of catheter angiography (CA). This observational trial analyzed all patients undergoing CA in 2006 and 2007 in one hospital. In 2007, patients having a low to intermediate cardiovascular risk and suspicion of coronary artery disease (CAD) and those with suspicion of progression of known organic heart disease (OHD) underwent CTCA either prior to CA or as the sole imaging modality. Appropriate utilization of CA was defined as: (1) percentage of patients showing normal or non-significant findings at CA, (2) percentage of self-referred patients to CA, and (3) percentage of patients with known $\mathrm{OHD}$ undergoing $\mathrm{CA}$ without immediate operative or interventional consequences. Use of CTCA resulted in a significant drop in the percentage of CA examinations in patients with suspected CAD showing normal or non-significant findings $(19 \%$ in $2006,10 \%$ in $2007, \mathrm{P}<$ $0.001)$. The percentage of self-referred CA significantly dropped $(29 \%$ in $2006,10 \%$ in $2007, \mathrm{P}<0.001)$. CT ruled-out CAD in 74/151 (49\%) patients, obviating subsequent CA. During a follow-up of $15+/-$ 4 months, CA and percutaneous interventions was considered necessary in 2/74 patients. CT ruled-out progression of known OHD in 53/60 (90\%) patients, while one patient underwent CA and percutaneous intervention during a follow-up period of $16+/-4$ months. No reduction of CA examinations without immediate consequences was found in patients with known OHD (13\% in 2006, 27\% in 2007). In patients with suspicion of CAD, CTCA improved the appropriate utilization of CA without jeopardizing patient safety, along with a decrease of self-referred patients for CA. CTCA did not influence the appropriate utilization of $\mathrm{CA}$ in patients with known OHD.
\end{abstract}

DOI: https://doi.org/10.1007/s10554-009-9541-3

Posted at the Zurich Open Repository and Archive, University of Zurich

ZORA URL: https://doi.org/10.5167/uzh-26611

Journal Article

Published Version

Originally published at:

Wagdi, P; Alkadhi, H (2010). The impact of cardiac CT on the appropriate utilization of catheter coronary angiography. International Journal of Cardiovascular Imaging, 26(3):333-344.

DOI: https://doi.org/10.1007/s10554-009-9541-3 


\title{
The impact of cardiac CT on the appropriate utilization of catheter coronary angiography
}

\author{
Philipp Wagdi · Hatem Alkadhi
}

Received: 13 February 2009/Accepted: 10 November 2009/Published online: 21 November 2009

(C) Springer Science+Business Media, B.V. 2009

\begin{abstract}
The purpose of this study was to evaluate the impact of computed tomography coronary angiography (CTCA) on the appropriate utilization of catheter angiography (CA). This observational trial analyzed all patients undergoing CA in 2006 and 2007 in one hospital. In 2007, patients having a low to intermediate cardiovascular risk and suspicion of coronary artery disease (CAD) and those with suspicion of progression of known organic heart disease (OHD) underwent CTCA either prior to CA or as the sole imaging modality. Appropriate utilization of CA was defined as: (1) percentage of patients showing normal or non-significant findings at CA, (2) percentage of selfreferred patients to $\mathrm{CA}$, and (3) percentage of patients with known OHD undergoing CA without immediate operative or interventional consequences. Use of CTCA resulted in a significant drop in the percentage of CA examinations in patients with suspected CAD showing normal or non-significant findings (19\% in 2006, $10 \%$ in 2007, $P<0.001$ ). The percentage of self-referred CA significantly dropped (29\% in 2006, $10 \%$ in $2007, P<0.001$ ). CT ruled-out CAD in $74 / 151$ (49\%) patients, obviating subsequent CA. During a follow-up of $15 \pm 4$ months, $\mathrm{CA}$ and percutaneous
\end{abstract}

P. Wagdi

Hirslanden Heart Center, Zurich, Switzerland

H. Alkadhi ( ()

Institute of Diagnostic Radiology, University Hospital

Zurich, Raemistr. 100, 8091 Zurich, Switzerland

e-mail: halkadhi@partners.org interventions was considered necessary in 2/74 patients. CT ruled-out progression of known OHD in 53/60 (90\%) patients, while one patient underwent CA and percutaneous intervention during a follow-up period of $16 \pm 4$ months. No reduction of CA examinations without immediate consequences was found in patients with known OHD (13\% in 2006, 27\% in 2007). In patients with suspicion of CAD, CTCA improved the appropriate utilization of CA without jeopardizing patient safety, along with a decrease of self-referred patients for CA. CTCA did not influence the appropriate utilization of CA in patients with known OHD.

Keywords Catheter angiography · Computed tomography · Coronary angiography . Appropriate utilization - Coronary artery disease

\section{Introduction}

The frequency of non-obstructive coronary artery disease (CAD) or negative findings at coronary catheter angiography (CA) has been proposed to be a surrogate marker for assessing the appropriate utilization of the invasive procedure [1-6]. The implicit assumption of this concept is that a high rate of nonsignificant disease or normal findings at CA might indicate an inappropriate use, which could reflect poor clinical judgment, inaccurate non-invasive studies, or 
possible self-interest [1]. Because the evaluation of the appropriate utilization of medical procedures is important to monitor the quality of care, to contain costs, and to enhance the safety by reducing inappropriate invasive procedures [7, 8], quality control (QC) criteria pertaining to the indication of CA have been defined [9]. Despite of these criteria, however, the incidence of normal or non-significant CA examinations has been reported to be around $30 \%[10,11]$, which has brought into question the appropriate utilization of the invasive procedure [1-6].

Multi-slice spiral computed tomography (CT) has recently emerged as a non-invasive tool for the anatomical imaging of the coronary arteries. The accuracy of CT coronary angiography (CTCA) for diagnosis or exclusion of CAD has been amply documented in various studies directly comparing CTCA with CA [12-15]. On the other hand, as with any new technologic innovation there is potential for misunderstanding and abuse. Despite the definition of appropriate utilization criteria for CTCA [16], concerns exist that the current boom of 64-slice and dualsource CT scanners and examinations could lead to unnecessary additional costs through an increase in the rate of $\mathrm{CA}$ in case of intermediate, non-diagnostic, or false positive CTCA examinations [17, 18]. Thus, over or misuse of cardiac CT could in turn have a negative effect on the appropriate utilization of CA.

In this study, we assessed the impact of clinically integrated CTCA with regard to the appropriate utilization of CA in patients with suspected CAD and in patients with known organic heart disease (OHD).

\section{Methods}

The study setting is a cardiology practice involving ambulatory non-invasive as well as invasive diagnosis, treatment, and consultancy in a private for-profit hospital, and the radiology institute of a public, nonprofit University Hospital. All consecutive patients undergoing $\mathrm{CA}$ and/or percutaneous interventions (PCI) during January 1st and December 31st in the years 2006 and 2007 were included in this retrospective study.

From the beginning of 2007 on, a modern CT scanner became available for cardiac imaging. Thus, during 2007 all patients presenting with chest pain and having a low or intermediate pre-test probability of $\mathrm{CAD}$, in whom the electrocardiogram (ECG) was equivocal or uninterpretable, and/or who were unable to exercise or delivered a submaximal stress test were subjected to CTCA, being in line with current recommendations [16]. In contrast, in the year 2006 all patients with similar symptoms, ECG and stress test findings directly underwent invasive work-up with CA.

In both years, all patients with chest pain and having a high pre-test probability of CAD were directly subjected to CA.

Patients presenting with or who were suspected of having an acute coronary syndrome were excluded from CTCA because the non-invasive modality is considered inappropriate for this indication [16]. Women below 40 years of age were also excluded from CTCA to keep radiation doses at a minimum because some of these patients eventually needed subsequent CA.

The protocol was approved by the local ethical committee who waived the informed consent requirement.

\section{Study population}

Patients undergoing CTCA were grouped as follows: those in which CAD was excluded, those in which a de-novo diagnosis of CAD was made, and those who underwent CTCA with previously documented OHD.

Patients undergoing CA were similarly grouped: those in which CAD was excluded, those in which CAD was diagnosed de-novo, and those with previously known OHD. OHD was defined to include coronary, valvular, or congenital heart disease, cardiomyopathy, or dysrhythmia requiring treatment (excluding sinus tachy- or bradycardia).

The appropriate utilization of CA was defined as previously shown [1-6]:

- The percentage of patients examined by CA with suspicion of de-novo CAD but showing no significant stenoses; and

- The proportion of patients without significant stenoses that were self-referred to CA by the examining cardiologist;

Appropriate utilization of CA was additionally extended to include:

- The percentage of patients with known OHD undergoing CA without immediate therapeutic 
consequences (e.g., percutaneous coronary intervention (PCI), coronary bypass surgery, valve replacement or repair, or pacemaker implantation).

In the further analysis, these three sub-groups were considered separately and were not summed-up to a single group.

\section{CT protocol and data reconstruction}

All patients were scanned on a dual-source CT scanner (Somatom Definition, Siemens Medical Solutions, Forchheim, Germany). An initial non-enhanced scan was performed for calcium scoring. Calcium scoring was performed for the purpose to give recommendations on the use of statins. Then, all patients received a single dose of $2.5 \mathrm{mg}$ isosorbiddinitrate s. 1. (Isoket, Schwarz Pharma, Monheim, Germany). No betablockers were given prior to CT in any patient. About $80 \mathrm{~mL}$ of iopromidum (Ultravist $370,370 \mathrm{mg} / \mathrm{mL}$, Bayer Schering Pharma, Berlin, Germany) was injected at a flow rate of $5 \mathrm{~mL} / \mathrm{s}$ followed by $30 \mathrm{~mL}$ saline solution. Contrast agent application was controlled by bolus-tracking in the ascending aorta (signal attenuation threshold $100 \mathrm{HU}$ ).

Scanning parameters were: detector collimation $2 \times 32 \times 0.6 \mathrm{~mm}$, slice collimation $2 \times 64 \times 0.6 \mathrm{~mm}$ by means of a z-flying focal spot, and gantry rotation time $330 \mathrm{~ms}$. In patients with a regular heart rate below 70 beats per minute (bpm), CT was performed with prospective electrocardiography (ECG)-gating and using attenuation-based tube current modulation (reference tube current time product: $190 \mathrm{mAs} /$ rotation). The data acquisition window was set at $70 \%$ of the RRinterval.

At heart rates above $70 \mathrm{bpm}$ or at irregular heart rates, CT was performed with retrospective ECGgating employing a heart rate-adapted pitch of $0.2-0.5$ and a tube current time product of $330 \mathrm{mAs} /$ rotation. ECG-pulsing for radiation dose reduction was used in all patients as previously recommended [19]. The tube potential was set at $120 \mathrm{kV}$ in patients with a body mass index (BMI) above $25 \mathrm{~kg} / \mathrm{m}^{2}$ and at $100 \mathrm{kV}$ in patients with a BMI below $25 \mathrm{~kg} / \mathrm{m}^{2}$. Both nonenhanced and contrast-enhanced CT scans were performed from the level of the tracheal bifurcation to the diaphragm in a cranio-caudal direction.

According to the dose-length products of the individual examinations (306-606 $\mathrm{mGy} \times \mathrm{cm}$ and
59-212 mGy $\times \mathrm{cm}$, respectively) and using a conversion coefficient of $0.017(\mathrm{mSv} /[\mathrm{mGy} \times \mathrm{cm}])$, the effective radiation doses of the retrospectively ECGgated protocols were $6.9 \pm 2.6 \mathrm{mSv}$ (range 5.2$10.3 \mathrm{mSv}$ ), and those of the prospectively ECG-gated protocols $2.2 \pm 0.6 \mathrm{mSv}$ (range 1.0-3.6 $\mathrm{mSv}$ ), being in line with previous reports [20,21].

Non-enhanced CT scans were reconstructed at $70 \%$ of the $\mathrm{R}-\mathrm{R}$ interval using $3.0 \mathrm{~mm}$ non-overlapping slices (reconstruction kernel B35f). Contrastenhanced CT scans were reconstructed within the RR-intervals of full tube current. CT images were reconstructed with a slice thickness of $0.75 \mathrm{~mm}$, a reconstruction increment of $0.5 \mathrm{~mm}$, and using a softtissue convolution kernel (B26f). In presence of vessel wall calcifications, additional images were reconstructed using a sharp-tissue convolution kernel (B46) to compensate for blooming artifacts.

\section{CT data analysis}

Coronary segments were defined according to a scheme proposed by the American Heart Association (AHA) [22]. The right coronary artery (RCA) was defined to include segments 1-4; the left main artery (LM) to consist of segment 5, the left anterior descending artery (LAD) to include segments 6-10, and the left circumflex artery (LCx) to include segments 11-15. The intermediate artery was designated as segment 16, if present, and considered to belong to the LAD.

All CTCA data analysis was performed during daily clinical practice by one experienced reader who was aware of the clinical history of the patients. Calcifications were quantified with scoring software (Syngo CaScore, Siemens). All lesions on more than two contiguous pixels with attenuation values greater than $130 \mathrm{HU}$ were marked and the calcium load in each patient was computed by using the Agatston method [23]. All coronary artery segments were assessed for the presence of significant stenoses, defined as luminal diameter narrowing $>50 \%$. Vessel diameters were measured on reconstructions perpendicularly oriented to the vessel centerline.

The report of each cardiac CT examination included the Agatston score, the age and gendermatched percentile of the Agatston score [24], the information about the presence or absence of coronary stenoses, and the \%-degree of stenoses, if 
present. The report was written at the day of each CT examination and was subsequently sent per mail to the referring cardiologist.

\section{Catheter angiography}

CA was performed according to standard techniques and at least three views in different planes were obtained for each coronary artery. The first author who was aware of the patients' clinical history and to the results from cardiac CT (which preceded CA in every patient) performed and evaluated all angiograms with regard to the presence (diameter reduction $>50 \%$ ) or absence of significant coronary stenoses. Coronary artery segments were defined according to the same AHA scheme as for CT [22]. All CA and $\mathrm{CT}$ examinations were performed within 4 weeks.

Clinical follow-up

All patients who were primarily excluded from CA but subjected to CTCA were strongly encouraged to undergo $\mathrm{CA}$ if chest pain persisted, or increased in duration, intensity, or frequency. This applied also to patients in whom the character of chest pain changed (for example if it became exclusively stress- or exercise-induced), and to those in whom an additional stress test performed within the follow-up time interval was positive for ischemia.

Mid-term follow-up information was obtained from all patients by either clinical visits or telephone interviews. The cardiologist discussed symptoms, the occurrence of any major adverse cardiac events (MACE) including myocardial infarction, heart failure, evolution of angina pectoris, coronary artery bypass graft surgery, PCI, or death, as well as hospital admission or repeated CA procedures.

\section{Statistical analysis}

Continuous variables were expressed as means \pm standard deviation (SD) and were compared using a paired $t$-test. Significance was assumed at a $P$ value $<0.05$. Categorical variables are reported as counts and percentages. Pearson correlation coefficients were computed for linear correlation analysis. Sensitivity, specificity, positive predictive value, and negative predictive value were calculated from Chi-Square tests of contingency, using the written reports of each CTCA examination, with $\mathrm{CA}$ as the standard of reference. Statistics for the diagnostic performance of CTCA were calculated on a per-vessel (i.e., at least one significant stenosis or absence of any significant stenosis in one coronary artery), and on a per-patient basis (i.e., at least one significant stenosis or absence of any significant stenosis per patient). All non-evaluative coronary segments at CTCA were considered as falsepositive findings on an intent-to-diagnose basis, because every patient with a non-evaluative segment would undergo CA in clinical practice, as previously shown [25]. All data were analyzed using commercially available statistical software (StatView 5.0; ASA Institute Inc., Cary, NC, USA).

\section{Results}

Year 2006

In 2006, a total number of 248 patients (81 female, mean age $67.3 \pm 10.3$ years, range $18-90$ years) underwent $\mathrm{CA}$ and/or PCI (excluding interventions in two patients for atrial septal defect (ASD) closure) (Fig. 1).

From these 248 patients, CAD could be excluded in $48(19.4 \%)$ (Table 1), CAD was diagnosed in 73 (29.4\%) (Table 2), and 127 patients (51.2\%) had known OHD (Table 3). In the group of patients showing no significant CAD, 14 (29\%) were selfreferred by the examining cardiologist. In the group of patients with known OHD, the CA examinations resulted in immediate therapeutic (i.e., interventional or surgical) consequences in 111 of the 127 patients $(87 \%)$. Forty-four of the patients $(35 \%)$ with known OHD were self-referred by the examining cardiologist.

Year 2007

In the year 2007, a total of 347 patients were included in the study (Fig. 1).

\section{CTCA}

From these 347 patients, 151 patients $(43.5 \%)$ were primarily referred to CTCA. CTCA was successfully performed in all of these 151 patients, and no procedure-related side-effects occurred. Fourty-four of the 151 patients (29\%) continued taking their 
Fig. 1 Patient management flow chart of the study

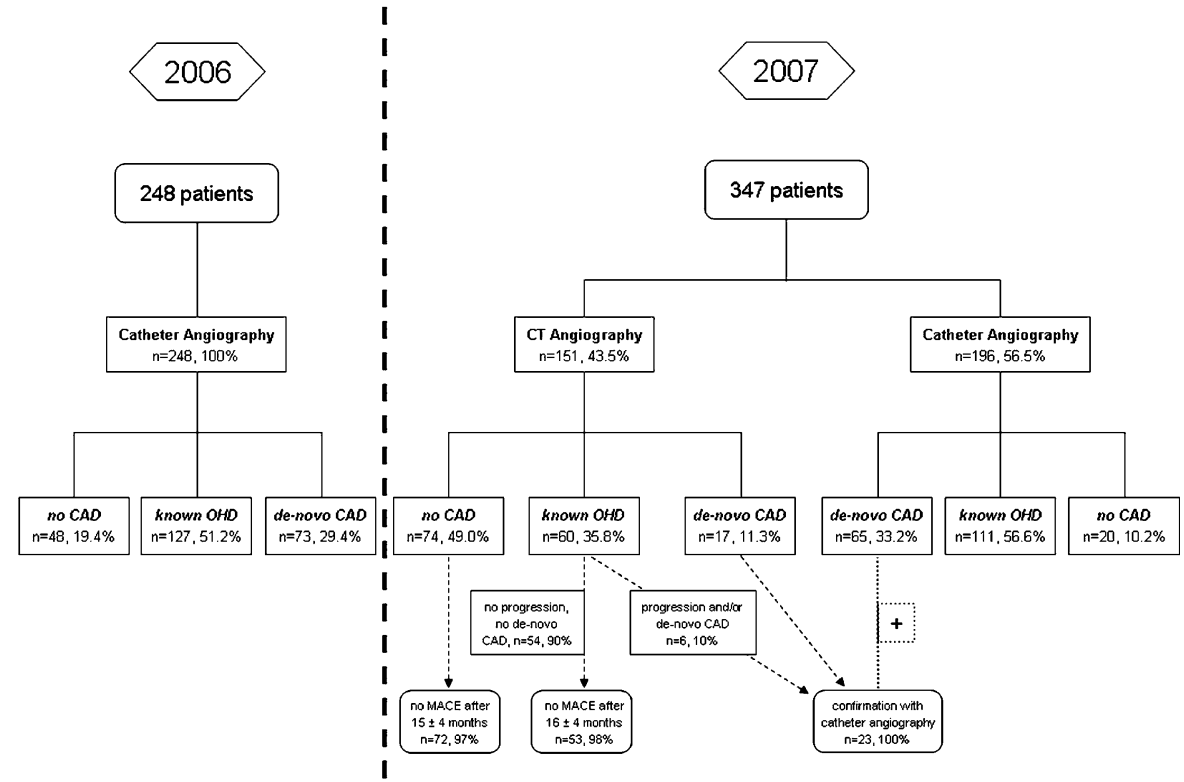

Table 1 Characteristics of patients undergoing catheter angiography showing no significant coronary artery disease in the years 2006 and 2007

\begin{tabular}{lll}
\hline & 2006 & 2007 \\
\hline Total number (females) & $48(28)$ & $20(13)$ \\
Age in years (range) & $63.5(37-90)$ & $60.9(40-82)$ \\
Referral pattern & & \\
EC & $14(29 \%)$ & $2(10 \%)$ \\
RC & $25(52 \%)$ & $13(65 \%)$ \\
RP & $8(17 \%)$ & $5(25 \%)$ \\
H & $1(2 \%)$ & - \\
Stress test positive & 8 & 5 \\
Negative & 11 & 6 \\
Borderline & 9 & 5 \\
Not done & 20 & 4 \\
CCS class & I: 8 & I: 1 \\
& II: 29 & II: 13 \\
& III: 11 & III: 6
\end{tabular}

CA coronary angiography, $C C S$ angina pectoris class according to Canadian Cardiovascular Society criteria, EC indication for CA by examining cardiologist, $H$ indication for CA by external hospital, $R C$ indication for CA by referring cardiologist, $R P$ indication for CA by referring physician

baseline beta-receptor antagonist medication at the time of CT, no additional medication for heart rate control was administered prior to the scans. The mean heart rate during CTCA was $67 \pm 15 \mathrm{bpm}$ (range 52-102 bpm). At CTCA, diagnostic image quality was found in $98.7 \%$ of all segments (2252/2282), while image quality of 30 segments $(1.3 \%$; RCA, $n=14$; LAD, $n=7$; LCx, $n=9$ ) was considered non-diagnostic and thus were rated as false-positive.

CTCA excluded significant CAD in 74 of the 151 patients $(49.0 \%$ ) (Table 4). CAD as a de-novo diagnosis was detected in 17 patients (11.3\%). In the 60 patients $(35.8 \%)$ with known OHD, progression of known CAD and/or de-novo CAD could be excluded in 54, while progression and/or de-novo CAD in those who were known to suffer from noncoronary cardiac disease was found in six patients (Table 5).

\section{Catheter angiography}

From the 347 patients studied in 2007, 196 (56.5\%) primarily underwent invasive work-up with CA (Fig. 1). Clinically suspected CAD was excluded by CA in 20 patients (10.2\%) (Table 1), 65 (33.2\%) were diagnosed to have a de-novo CAD (Table 2), whereas $111(56.6 \%)$ suffered from known OHD (Table 3).

CA was performed additionally in 23 patients after obtaining the results from CTCA (including 17 patients with a de-novo diagnosis of $\mathrm{CAD}$ and 6 with a progression of known OHD), leading to a total number of 219 CA examinations in 2007 (being significantly less than in 2006, $P<0.0001$ ). 
Table 2 Characteristics of patients undergoing catheter angiography showing significant coronary artery disease as a de-novo diagnosis

\begin{tabular}{lll}
\hline & 2006 & 2007 \\
\hline Total number (female) & $73(14)$ & $65^{\mathrm{a}}(30)$ \\
Age in years (range) & $67.4 \pm 10.1$ & $68.0 \pm 10.4$ \\
& $(39-88)$ & $(37-87)$ \\
Referral pattern & & \\
EC & $16(22 \%)$ & $40(46 \%)$ \\
RC & $29(40 \%)$ & $22(25 \%)$ \\
RP & $22(30 \%)$ & $20(23 \%)$ \\
H & $6(8 \%)$ & $5(6 \%)$ \\
Treatment & & \\
PCI & $45(62 \%)$ & $48(55 \%)$ \\
ACB & $20(27 \%)$ & $25(29 \%)$ \\
Conservative & $8(11 \%)$ & $12(14 \%)$ \\
Other (PTA, AVA/ & - & $2(2 \%)$ \\
PM) & & \\
\hline
\end{tabular}

$A C B$ Aorto-coronary bypass surgery, $E C$ indication for CA by examining cardiologist, $H$ indication for $\mathrm{CA}$ by external hospital, PCI Percutaneous coronary intervention, PTA peripheral angioplasty, $R C$ indication for $\mathrm{CA}$ by referring cardiologist, $R P$ indication for CA by referring physician, $A V A$ / $P M$ AV node ablation and pacemaker implantation. Only the main intervention is indicated, some patients underwent more than one procedure (e.g., PCI and PTA)

${ }^{\text {a }}$ The 23 patients with a de-novo diagnosis of CAD and those with a progression of known OHD made with CT in 2007 are excluded

Comparison between 2006 and 2007

The number of patients undergoing CA but having no CAD significantly $(P<0.0001)$ decreased in 2007 as compared to 2006 (20 patients representing $10.2 \%$ of those 196 undergoing CA in 2007 versus 48 patients representing $19.4 \%$ of the 248 undergoing CA in 2006, Table 1).

Similarly, the number of self-referrals in this patient group significantly $(P<0.0001)$ decreased in 2007 (2 representing $10 \%$ versus 14 representing $29 \%$ of the patients undergoing CA in 2007 and 2006, Table 1). In the same period of time, the proportion of patients referred to CA by external doctors (including external hospital, referring cardiologist, and referring physician) significantly increased $(P<0.001)$, because CTCA was not used as a filter test in these patients (i.e., the patients were directly referred to invasive work-up).

The percentage of patients with a de-novo diagnosis of $\mathrm{CAD}$ at $\mathrm{CA}$ that was followed by an immediate
Table 3 Characteristics of patients undergoing catheter angiography with known organic heart disease

\begin{tabular}{lcc}
\hline & 2006 & 2007 \\
\hline Total number (female) & $127(39)$ & $111(32)$ \\
Age in years (range) & $66.4 \pm 10.6$ & $67.6 \pm 12.0$ \\
& $(32-89)$ & $(26-92)$ \\
Referral pattern & & \\
EC & $44(35 \%)$ & $40(36 \%)$ \\
RC & $47(37 \%)$ & $39(35 \%)$ \\
RP & $20(16 \%)$ & $29(26 \%)$ \\
H & $16(13 \%)$ & $4(4 \%)$ \\
Treatment & & \\
PCI & $50(39 \%)$ & $37(33 \%)$ \\
ACB* & $11(9 \%)$ & $8(7 \%)$ \\
Conservative & $16(13 \%)$ & $30(27 \%)$ \\
Other (AVA/PM, PTA, & $36(28 \%)$ & $18(16 \%)$ \\
VS)** & & \\
PFO/ASD-C & $9(8 \%)$ & $19(17 \%)$ \\
\hline
\end{tabular}

$A C B$ Aortocoronary bypass, AVA/PM AV node ablation and pacemaker insertion, $C A$ coronary angiography, $E C$ examining cardiologist, $H$ Hospital, $P C I$ percutaneous coronary intervention, $P T A$ percutaneous angioplasty of peripheral or renal artery, $R C$ referring cardiologist, $R P$ referring physician, $V S$ valvular surgery

* Some patients underwent combined ACB and VS

** Including aortic aneurysm surgery

therapeutic consequence decreased significantly $(P<0.05)$ from $89 \%$ in 2006 to $84 \%$ in 2007 , owing mainly to a decrease in PCI procedures (Table 2).

In patients with known OHD, the rate of therapeutic procedures after invasive diagnosis significantly $(P<0.0001)$ decreased from $87 \%$ in 2006 to $73 \%$ in 2007 (Table 3). During the same period of time, the proportion of patients with known OHD who were self-referred to CA by the author remained unchanged (35 vs. $36 \%, P=$ n.s.).

\section{Comparison between CTCA and CA}

Twenty-three patients underwent CA after a de-novo diagnosis of CAD or a progression of known CAD at CTCA (Fig. 2). In these patients, the sensitivity, specificity, positive and negative predictive value of CTCA was $100 \%$ each in the per-patient based analysis, with lower values in the per-vessel based analysis (Table 6). The correlation regarding the percent stenosis estimation between CTCA and CA 
Table 4 Characteristics of patients in whom significant coronary artery disease was excluded with CT

\begin{tabular}{|c|c|}
\hline & $N=74$ \\
\hline Age in years (range) & $62 \pm 11(37-87)$ \\
\hline Gender & 41 females \\
\hline CVRF & $\begin{array}{l}\text { Positive family history: } 42 \\
\quad(57 \%) \\
\text { Hyperlipidemia } 43(58 \%) \\
\text { Smoking } 28(38 \%) \\
\text { Hypertension } 35(47 \%) \\
\text { Diabetes mellitus } 3(4 \%)\end{array}$ \\
\hline Symptoms* & $\begin{array}{l}\text { Chest pain } 53(72 \%) \\
\text { Dyspnea } 7(9) \\
\text { None } 16(22) \\
\text { Syncope } 1(1.4 \%) \\
\text { PE } 1(1.4 \%) \\
\text { CVI } 1(1.4 \%)\end{array}$ \\
\hline Stress test & $\begin{array}{l}\text { Negative } 32(43 \%) \\
\text { SM } 29(39 \%) \\
\text { Borderline } 6(8 \%) \\
\text { Positive } 4(5 \%) \\
\text { None } 1(1 \%) \\
\text { BBB } 2(3 \%)\end{array}$ \\
\hline $\begin{array}{l}\text { Agatston score according } \\
\text { to age and gender-matched } \\
\text { percentile [24] }\end{array}$ & $\begin{array}{l}\text { Between 0-25\%: } 32 \\
\text { Between 25-50\%: } 9 \\
\text { Between 50-75\%: } 10 \\
\text { Between 75-90\%: } 15 \\
\text { Above 90\%: } 8\end{array}$ \\
\hline$\%$ stenosis at CT (range) & $\begin{array}{l}\text { LAD } 7 \% \pm 15(0-50 \%) \\
\mathrm{LCx} 4 \% \pm 12(0-50 \%) \\
\operatorname{RCA} 4 \% \pm 11(0-50 \%)\end{array}$ \\
\hline MACE & $2 ; 2.7 \%(\mathrm{CA}$ and $\mathrm{PCI})$ \\
\hline $\begin{array}{l}\text { Follow-up time in months } \\
\text { (range) }\end{array}$ & $15.2 \pm 3.7(12-24)$ \\
\hline
\end{tabular}

$B B B$ bundle branch block, $C V I$ cerebrovascular insult, $C V R F$ cardiovascular risk factors, $L A D$ left anterior descending artery, $P E$ pulmonary edema during competition sport, $R C A$ right coronary artery, $L C x$ left circumflex artery, $S M$ clearly submaximal work load at stress test due to deconditioning or invalidating knee, hip or back pain (mostly preoperative screening), MACE major adverse cardiac events

* More than one symptom or none possible

was significant $(P<0.001)$, with highest correlation coefficients for the RCA $(r=0.92)$, followed by the LAD $(r=0.84)$ and the LCx $(r=0.77)$.
Table 5 Characteristics of patients with known organic heart disease undergoing CT

\begin{tabular}{|c|c|}
\hline & $N=60^{\mathrm{a}}$ \\
\hline Age in years (range) & $70 \pm 9.7(40-87)$ \\
\hline Gender & 17 Females \\
\hline \multirow[t]{5}{*}{ CVRF } & Positive family history $39(72 \%)$ \\
\hline & Hyperlipidemia 44 (81\%) \\
\hline & Smoking $18(33 \%)$ \\
\hline & Hypertension 38 (70\%) \\
\hline & Diabetes mellitus $6(11 \%)$ \\
\hline \multirow{9}{*}{$\begin{array}{l}\text { CAD or organic heart } \\
\text { disease known prior } \\
\text { to } \mathrm{CT}\end{array}$} & CAD post bypass $9(17 \%)$ \\
\hline & CAD post-PCI $25(46 \%)$ \\
\hline & CAD* $8(15 \%)$ \\
\hline & Valvular heart disease $3(6 \%)$ \\
\hline & ASD $2(4 \%)$ \\
\hline & Cardiomyopathy 2 (4\%) \\
\hline & Atrial fibrillation $3(6 \%)$ \\
\hline & Cor pulmonale $1(2 \%)$ \\
\hline & $\begin{array}{l}\text { Coronary anomaly (left main from } \\
\text { right coronary) } 1(2 \%)\end{array}$ \\
\hline \multirow[t]{5}{*}{ Stress test } & None $13(24 \%)$ \\
\hline & Negative $23(43 \%)$ \\
\hline & Submaximal $8(15 \%)$ \\
\hline & Positive $6(11 \%)$ \\
\hline & Borderline $4(7 \%)$ \\
\hline MACE & $1 ; 1.7 \%(\mathrm{CA}$ and PCI) \\
\hline $\begin{array}{l}\text { Follow-up time in months } \\
\text { (range) }\end{array}$ & $16.1 \pm 4.2(12-24)$ \\
\hline Interventions & ASD closure in 2 patients \\
\hline
\end{tabular}

MACE major adverse cardiac events, $C A D$ coronary artery disease, $A S D$ atrial septal defect

* Patients with a history of myocardial infarction or maximally a $60 \%$ stenosis and no PCI

${ }^{a}$ Progression of CAD or de-novo CAD in patients with known non-coronary cardiac disease was ruled-out with $\mathrm{CT}$ in 54 patients in whom no subsequent catheter angiography was performed. Six patients showed progression of disease and underwent subsequent catheter angiography

\section{Clinical follow-up}

From the 74 patients in whom significant stenoses were ruled-out at CTCA, 72/74 had no MACE in the mid-term follow-up period of $15.2 \pm 3.7$ months (range 12-24 months). Two of the patients with normal CTCA had recurrent angina necessitating CA that revealed a significant stenosis of the LCx and an in-stent restenosis of the LAD, respectively. 

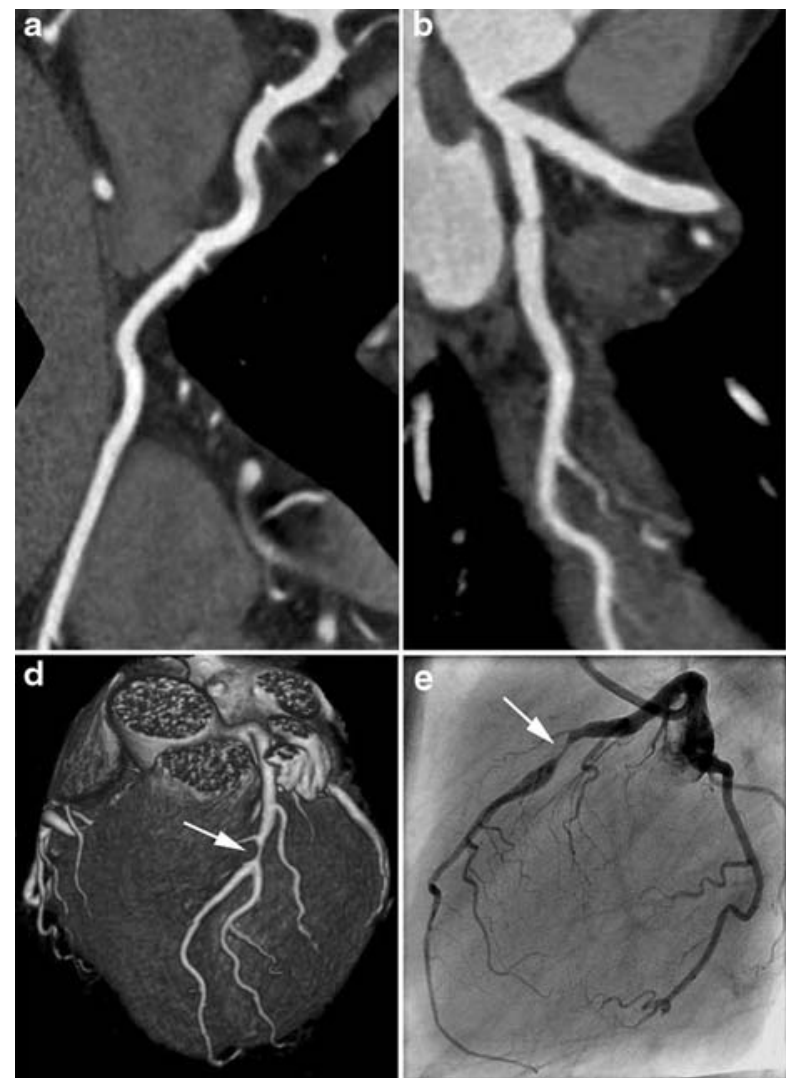

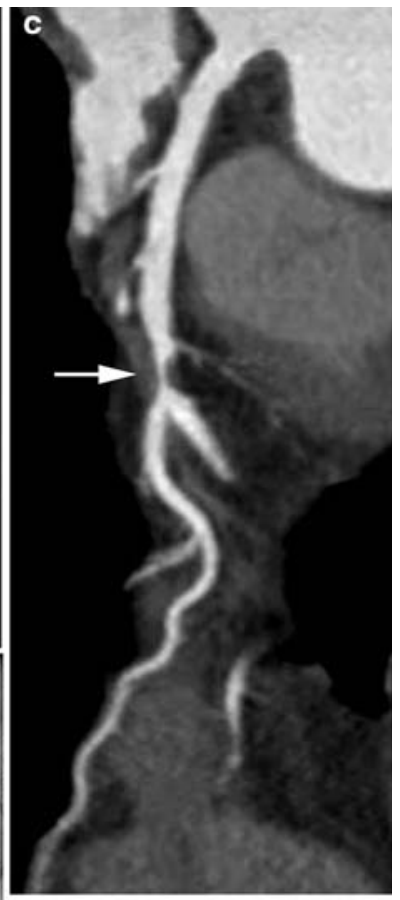

soft plaque is shown (arrows). The stenoses was subsequently confirmed at catheter angiography (e, arrow), with subsequent successful angioplasty and stenting (not shown)

All patients having an intermediate risk of cardiovascular disease and Agatston scores above the 75th age- and gender-matched percentile [24] as well as having non-obstructive $\mathrm{CAD}$ were selected for more aggressive target values for lipid-lowering therapy (i.e., statins) and were advised to take salicylic acid medication, as previously recommended [26].

\section{Discussion}

This observational study has been intended to investigate the role of cardiac $\mathrm{CT}$ on the appropriate utilization of CA. By virtue of its design, the study has four major strengths. First, it represents-to the best of our knowledge-the first investigation exploring the usefulness, efficiency, and safety of the incorporation of non-invasive coronary angiography 
Table 6 Per-vessel and per-patient based analysis of the diagnostic performance of dual-source CT compared to CA for the identification of significant $(>50 \%)$ coronary stenosis in the 23 patients who underwent both modalities

\begin{tabular}{lrlcl}
\hline Number of patients =23 & LAD & LCx & RCA & Patients* \\
Stenoses correctly & $13 / 14$ & $5 / 7$ & $7 / 9$ & $20 / 20^{*}$ \\
$\quad$ identified (\%) & & & & \\
Stenoses missed (\%) & $1 / 14$ & $2 / 7$ & $2 / 9$ & $0 / 20^{*}$ \\
Stenoses incorrectly & 0 & 1 & 0 & $0 / 20^{*}$ \\
$\quad$ diagnosed (\%) & & & & \\
Sensitivity (\%) & 93 & 71 & 78 & 100 \\
Specificity (\%) & 100 & 94 & 100 & 100 \\
Positive predictive value (\%) & 100 & 83 & 100 & 100 \\
Negative predictive value $(\%)$ & 90 & 88 & 88 & 100 \\
\hline
\end{tabular}

$L A D$ left anterior descending, $R C A$ right coronary artery, $L C x$ left circumflex artery

* 20/23 patients had significant stenosis of at least one main vessel (LAD, LCx, RCA). Three patients had significant stenosis of a diagonal branch, two of which were correctly identified by $\mathrm{CT}$, one was missed, the patient had persistent typical symptoms and thus underwent CA. Two stenoses at posterolateral branches of the $\mathrm{LCx}$ were missed at CT

with CT into daily clinical practice. Second, the study excludes any bias due to conflicts of interest. The two persons and institutions involved played a complementary role in this study although in general being competitors (i.e., one working in a public non-profit, the other in a private for-profit institution). Third, it would be in the very financial interest of one of the authors (the referring cardiologist) to perform as many coronary angiographies as possible, whereas the other author (the interpreting radiologist) is being salaried with no financial incentive to increase the numbers of CT examinations. Fourth, we have explicitly applied our measures of appropriate utilization to a small unit of observation at the level of two centers and physicians, as previously suggested $[6,27]$.

In this study, we used the known QC criteria percentage of patients undergoing $\mathrm{CA}$ but having no or non-significant CAD and the percentage of selfreferred patients for testing the appropriate utilization of CA [1-6]. We extended these QC criteria by considering also the percentage of patients with known OHD undergoing $\mathrm{CA}$ without interventional or surgical consequences.

By doing so, our study shows that the integration of CTCA into the clinical patient management helps to rationalize the use of CA in patients referred with a

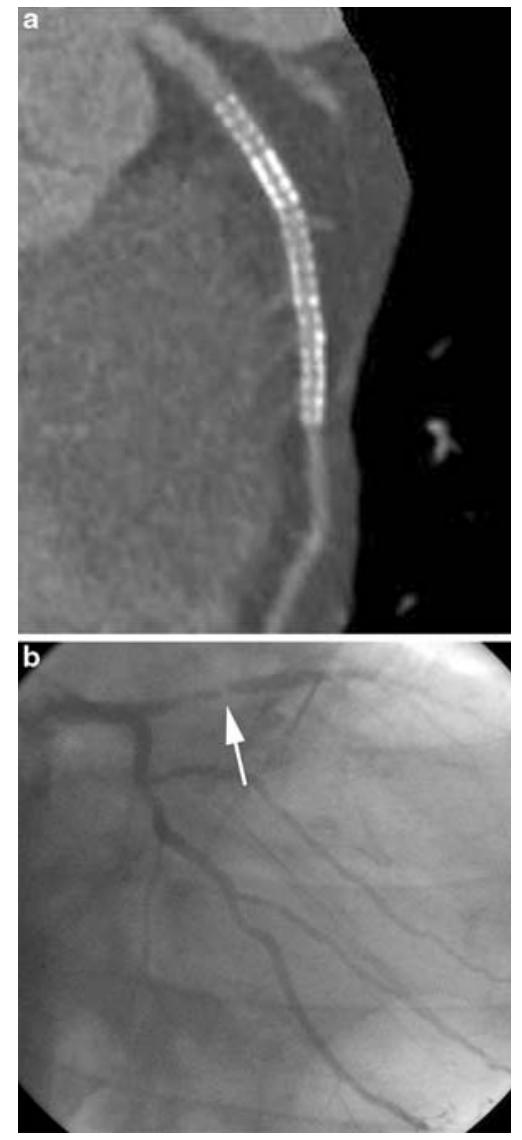

Fig. 3 A 59-year-old male patient. Curved multi-planar reformations of the left anterior descending artery showing patent stents in the proximal and mid-segment (a). CA performed 8 months later due to recurrent chest pain revealed a high-grade in-stent restenosis in the proximal segment (arrow, b)

clinical suspicion of CAD. In this group, substantially less patients showing normal or non-significant $\mathrm{CAD}$ underwent $\mathrm{CA}$, and less of these patients were selfreferred by the examining cardiologist. This could be achieved through the use of CTCA as a filter test while subjecting those patients to the non-invasive modality who had chest pain and a low to intermediate cardiovascular risk profile, an equivocal or uninterpretable ECG, and/or who were unable to exercise in a sufficient manner. Interestingly, this group of patients exactly represents that population that has been recommended by various international societies to benefit most from non-invasive coronary angiography with CT [16, 26, 28].

The incidence of morbidity and mortality associated with $\mathrm{CA}$ is low, however, not negligible. 

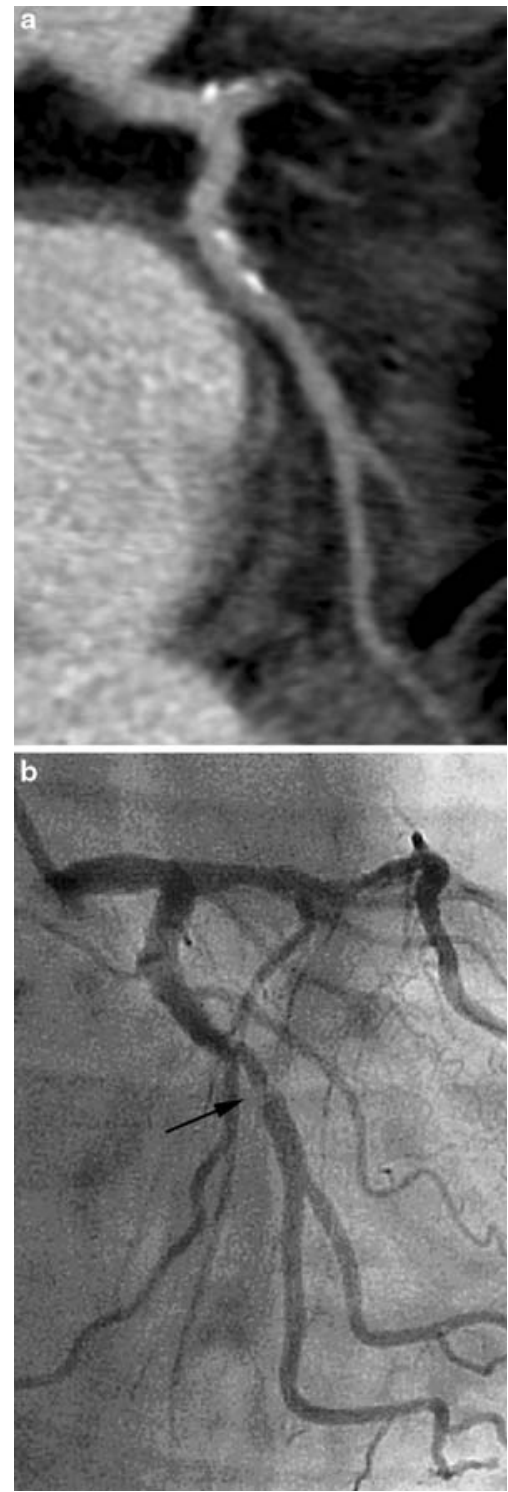

Fig. 4 A 65-year-old male patient. Curved multi-planar reformations of the left circumflex artery shows vessel wall irregularities but no significant stenosis (a). CA performed 6 months later shows a high-grade stenoses (arrow) in the proximal segment (b)

According to several studies, CA implies risks to the patient through a major complication rate of up to $1.7 \%$ [29]. Thus, the use of CA should be performed in accordance with current guidelines, such as those of the American College of Cardiology and American Heart Association [9]. Nevertheless-and notwithstanding the use of these guidelines-normal or non-significant $\mathrm{CA}$ examinations in such a population remain [30] and expose the patients to the risk of a potentially inappropriate invasive procedure. Our study demonstrates that CTCA can be used as a safe filter test either as an alternative to $\mathrm{CA}$ in patients showing no $\mathrm{CAD}$, or as an anatomical imaging modality rationalizing the use of invasive work-up with CA.

During the follow-up period up of this study, two of 74 patients in the group examined by CTCA for de-novo $\mathrm{CAD}$ and one of 54 patients from the group with known OHD underwent CA and PCI. These three patients suffered from recurrent angina more than 8 months after CTCA, and none of these patients suffered from myocardial infarction, cerebrovascular event, heart failure or death. Retrospective analysis of the CTCA examinations in these three patients confirmed the initial report. Thus, progression of CAD may have played a role in these patients.

Many unnecessary CA examinations are performed in patients having some OHD of no prognostic significance (e.g., patients with atypical symptoms and no ischemia that had undergone a PCI or ACB surgery in the past, or patients with mild to moderate valvular heart disease in which stress testing is equivocal). Use of CTCA as a filter test in these patients did not decrease the number of inappropriate CA examinations (defined as invasive work-up without therapeutic consequences). In contrast, the percentage of patients not undergoing any intervention after CA increased. The cause of this finding either reflects a chance effect when dealing with a numerically small population or may reflect a selection bias, i.e., a lower threshold of indication for CA in patients with known OHD. This must be weighed against the benefit for patients with known OHD who were investigated only by CTCA that safely documented the absence of progression of known CAD and/or de-novo CAD in those with known non-coronary cardiac disease.

In Switzerland, CA is more expensive than cardiac CT by a factor of 5- to 12-fold (the latter in persons with a private health insurance). Thus, the costs for the 74 patients where CTCA ruled-out CAD added to the costs of the 54 patients where progression of CAD and/or de-novo CAD in patients with OHD was ruled-out with CTCA must be weighed against the costs in the 23 patients who underwent both invasive and non-invasive work-up.

The effective radiation doses of our CTCA protocols ranged between 1 and $10 \mathrm{mSV}$, depending on the 
protocol used. These values must be opposed to those of diagnostic CA examinations that have been reported to range between 2 and $23 \mathrm{mSv}$ [31]. Similar to the financial aspects mentioned above, the cumulative radiation doses in the 23 patients undergoing both anatomic imaging modalities must be weighted against the 128 patients who only underwent CTCA.

In the 23 patients undergoing both CTCA and CA, the correlation regarding the degree of stenoses was good, with similar results as in previous studies [13, 32]. The overall diagnostic performance for the identification of patients having significant stenoses was excellent and corroborates with previous studies comparing the accuracy of CTCA with CA [12-15]. It must be noted, however, that the subgroup of patients available for this comparison is biased through the inclusion of only those patients showing significant stenoses at CTCA and by the knowledge of the CT findings by the examining cardiologist performing CA.

\section{Study limitations}

The study reports only the practice of a single center low-volume cardiology practice (248, respectively, 219 invasive diagnostic procedures in 2006 and 2007 , and 95, respectively, 85 PCI procedures, in addition to other interventions). On the other hand, this may reflect the practice of a substantial number of cardiologists working in non-academic settings, both in the U.S. and in Europe and has been suggested to be the optimal size for such observations $[6,27]$. Second, the term "significance" when using small numbers must be interpreted with caution. Third, the follow-up period in our study was only approximately one and a half years, and no long-term risk prediction in our patients undergoing CT is available. Finally, we did not use the most modern CT scanner technology and protocols [33-35] which may have further lowered the radiation exposure to our patients.

\section{Conclusions}

In patients with chest pain having a low- to intermediate risk for CAD and an equivocal or uninterpretable ECG, and/or who are unable to exercise in a sufficient manner, CTCA can be used as a test that helps to rationalize the use of $\mathrm{CA}$ without putting a risk on the mid-term outcome of the patients. Implementing cardiac CT into clinical routine results in an improved appropriate utilization of CA examinations through a reduction of purely diagnostic invasive work-up and at the same time reduces the rate of self-referred patients. In patients with known OHD, in contrast, the use of cardiac CT proved not to enhance the appropriate utilization of CA procedures because the rate of invasive work-up without therapeutic consequences was not reduced.

Acknowledgments This paper is dedicated by P.W. to the memory of G. Marquardt. H.A. is supported by the National Center of Competence in Research, Computer Aided and Image Guided Medical Interventions of the Swiss National Science Foundation. He is completely independent from the funders.

Conflicts of interest statement No conflicts of interest.

\section{References}

1. Farrehi PM, Bernstein SJ, Rasak M et al (2002) Frequency of negative coronary arteriographic findings in patients with chest pain is related to community practice patterns. Am J Manag Care 8:643-648

2. Bernstein SJ, Hilborne LH, Leape LL, Park RE, Brook RH (1994) The appropriateness of use of cardiovascular procedures in women and men. Arch Intern Med 154:2759-2765

3. Brook RH, Park RE, Chassin MR, Solomon DH, Keesey J, Kosecoff J (1990) Predicting the appropriate use of carotid endarterectomy, upper gastrointestinal endoscopy, and coronary angiography. N Engl J Med 323:1173-1177

4. Leape LL, Park RE, Solomon DH, Chassin MR, Kosecoff J, Brook RH (1990) Does inappropriate use explain small-area variations in the use of health care services? Jama 263: 669-672

5. Noonan SJ, Cook JL, Keller CE et al (1995) Relationship of anatomic disease to appropriateness ratings of coronary angiography. Arch Intern Med 155:1209-1213

6. Phelps CE (1993) The methodologic foundations of studies of the appropriateness of medical care. N Engl J Med 329: 1241-1245

7. Ayanian JZ, Landrum MB, Normand SL, Guadagnoli E, McNeil BJ (1998) Rating the appropriateness of coronary angiography-do practicing physicians agree with an expert panel and with each other? N Engl J Med 338:1896-1904

8. Shekelle PG (2001) Are appropriateness criteria ready for use in clinical practice? N Engl J Med 344:677-678

9. Bashore TM, Bates ER, Berger PB et al (2001) American college of cardiology/society for cardiac angiography and interventions clinical expert consensus document on cardiac 
catheterization laboratory standards. A report of the American college of cardiology task force on clinical expert consensus documents. J Am Coll Cardiol 37:2170-2214

10. Faxon DP, McCabe CH, Kreigel DE, Ryan TJ (1982) Therapeutic and economic value of a normal coronary angiogram. Am J Med 73:500-505

11. Phibbs B, Fleming T, Ewy GA et al (1988) Frequency of normal coronary arteriograms in three academic medical centers and one community hospital. Am J Cardiol 62: 472-474

12. Leschka S, Alkadhi H, Plass A et al (2005) Accuracy of MSCT coronary angiography with 64-slice technology: first experience. Eur Heart J 26:1482-1487

13. Raff GL, Gallagher MJ, O’Neill WW, Goldstein JA (2005) Diagnostic accuracy of noninvasive coronary angiography using 64-slice spiral computed tomography. J Am Coll Cardiol 46:552-557

14. Mollet NR, Cademartiri F, van Mieghem CA et al (2005) High-resolution spiral computed tomography coronary angiography in patients referred for diagnostic conventional coronary angiography. Circulation 112:2318-2323

15. Ropers U, Ropers D, Pflederer T et al (2007) Influence of heart rate on the diagnostic accuracy of dual-source computed tomography coronary angiography. J Am Coll Cardiol 50:2393-2398

16. Hendel RC, Patel MR, Kramer CM et al (2006) ACCF/ACR/ SCCT/SCMR/ASNC/NASCI/SCAI/SIR 2006 appropriateness criteria for cardiac computed tomography and cardiac magnetic resonance imaging: a report of the American College of Cardiology Foundation Quality Strategic Directions Committee Appropriateness Criteria Working Group, American College of Radiology, Society of Cardiovascular Computed Tomography, Society for Cardiovascular Magnetic Resonance, American Society of Nuclear Cardiology, North American Society for Cardiac Imaging, Society for Cardiovascular Angiography and Interventions, and Society of Interventional Radiology. J Am Coll Cardiol 48:1475-1497

17. Mitka M (2006) CT angiography: clearer picture, Fuzzier reception. Jama 295:1989-1990

18. Wann S, Nassef AL, Jeffrey J et al (2007) Ethical considerations in CT angiography. Int J Cardiovasc Imaging 23:379-388

19. Leschka S, Scheffel H, Desbiolles L et al (2007) Image quality and reconstruction intervals of dual-source CT coronary angiography: recommendations for ECG-pulsing windowing. Invest Radiol 42:543-549

20. Leschka S, Stolzmann P, Schmid FT et al (2008) Low kilovoltage cardiac dual-source CT: attenuation, noise, and radiation dose. Eur Radiol 18:1809-1817

21. Stolzmann P, Scheffel H, Schertler T et al (2008) Radiation dose estimates in dual-source computed tomography coronary angiography. Eur Radiol 18:592-599

22. Austen WG, Edwards JE, Frye RL et al (1975) A reporting system on patients evaluated for coronary artery disease. Report of the ad hoc Committee for Grading of Coronary
Artery Disease, Council on Cardiovascular Surgery, American Heart Association. Circulation 51:5-40

23. Agatston AS, Janowitz WR, Hildner FJ, Zusmer NR, Viamonte M Jr, Detrano R (1990) Quantification of coronary artery calcium using ultrafast computed tomography. J Am Coll Cardiol 15:827-832

24. Hoff JA, Chomka EV, Krainik AJ, Daviglus M, Rich S, Kondos GT (2001) Age and gender distributions of coronary artery calcium detected by electron beam tomography in 35, 246 adults. Am J Cardiol 87:1335-1339

25. Alkadhi H, Scheffel H, Desbiolles L et al (2008) Dualsource computed tomography coronary angiography: influence of obesity, calcium load, and heart rate on diagnostic accuracy. Eur Heart J 29:766-776

26. Budoff MJ, Achenbach S, Blumenthal RS et al (2006) Assessment of coronary artery disease by cardiac computed tomography: a scientific statement from the American Heart Association Committee on Cardiovascular Imaging and Intervention, Council on Cardiovascular Radiology and Intervention, and Committee on Cardiac Imaging, Council on Clinical Cardiology. Circulation 114:1761-1791

27. Brook RH (1989) Practice guidelines and practicing medicine. Are they compatible? Jama 262:3027-3030

28. Fox K, Garcia MA, Ardissino D et al (2006) Guidelines on the management of stable angina pectoris: executive summary: the Task Force on the Management of Stable Angina Pectoris of the European Society of Cardiology. Eur Heart J 27:1341-1381

29. Zanzonico P, Rothenberg LN, Strauss HW (2006) Radiation exposure of computed tomography and direct intracoronary angiography: risk has its reward. J Am Coll Cardiol 47: 1846-1849

30. Gilard M, Le Gal G, Cornily JC et al (2007) Midterm prognosis of patients with suspected coronary artery disease and normal multislice computed tomographic findings: a prospective management outcome study. Arch Intern Med 167:1686-1689

31. Einstein AJ, Moser KW, Thompson RC, Cerqueira MD, Henzlova MJ (2007) Radiation dose to patients from cardiac diagnostic imaging. Circulation 116:1290-1305

32. Leber AW, Knez A, von Ziegler F et al (2005) Quantification of obstructive and nonobstructive coronary lesions by 64-slice computed tomography: a comparative study with quantitative coronary angiography and intravascular ultrasound. J Am Coll Cardiol 46:147-154

33. Rybicki FJ, Otero HJ, Steigner ML et al (2008) Initial evaluation of coronary images from 320-detector row computed tomography. Int $\mathrm{J}$ Cardiovasc Imaging 24: 535-546

34. Achenbach S, Marwan M, Schepis T et al (2009) Highpitch spiral acquisition: a new scan mode for coronary CT angiography. J Cardiovasc Comput Tomogr 3:117-121

35. Leschka S, Stolzmann P, Desbiolles L, et al. (2009) Diagnostic accuracy of high-pitch dual-source CT for the assessment of coronary stenoses: first experience. Eur Radiol. doi:10.1007/s00330-009-1618-9 